\title{
Identification of an interaction between calcium-dependent protein kinase 4 (EtCDPK4) and serine protease inhibitor (EtSerpin) in Eimeria tenella
}

Ling Lv ${ }^{1,2+}$, Bing Huang ${ }^{1,2+}$, Qiping Zhao², Zongping Zhao², Hui Dong ${ }^{2}$, Shunhai Zhu², Ting Chen², Ming Yan ${ }^{1,2}$ and Hongyu Han ${ }^{2^{*}}$

\begin{abstract}
Background: Eimeria tenella is an obligate intracellular apicomplexan protozoan parasite that has a complex lifecycle. Calcium ions, through various calcium-dependent protein kinases (CDPKs), regulate key events in parasite growth and development, including protein secretion, movement, differentiation, and invasion of and escape from host cells. In this study, we identified proteins that interact with EtCDPK4 to lay a foundation for clarifying the role of CDPKs in calcium channels.

Methods: Eimeria tenella merozoites were collected to construct a yeast two-hybrid $(\mathrm{Y} 2 \mathrm{H}) \mathrm{CDNA}$ library. The $\mathrm{Y} 2 \mathrm{H}$ system was used to identify proteins that interact with EtCDPK4. One of interacting proteins was confirmed using bimolecular fluorescence complementation and co-immunoprecipitation in vivo. Co-localization of proteins was performed using immunofluorescence assays.

Results: Eight proteins that interact with EtCDPK4 were identified using the $\mathrm{Y} 2 \mathrm{H}$ system. One of the proteins, $E$. tenella serine protease inhibitor 1 (EtSerpin), was further confirmed.

Conclusion: In this study, we screened for proteins that interact with EtCDPK4. An interaction between EtSerpin and EtCDPK4 was identified that may contribute to the invasion and development of E. tenella in host cells.

Keywords: Eimeria tenella, Calcium-dependent protein kinases, Serpin, Yeast two-hybrid, Bimolecular fluorescence complementation, Co-immunoprecipitation
\end{abstract}

\section{Background}

Eimeria tenella is an obligate intracellular apicomplexan protozoan parasite that causes huge economic losses in the poultry industry. Protozoans have complex lifecycles and need to invade host cells to grow, develop and reproduce. Invasion is a multi-step process that involves the formation, in most cases, of parasitophorous vacuoles within the host cells in which the parasites replicate [1]. Successful invasion of and subsequent escape from host cells, as well as spreading within the host, are

\footnotetext{
* Correspondence: hhysh@shvri.ac.cn

${ }^{\dagger}$ Equal contributors

${ }^{2}$ Shanghai Veterinary Research Institute, Chinese Academy of Agricultural Sciences, Key Laboratory of Animal Parasitology of Ministry of Agriculture, Minhang, Shanghai 200241, People's Republic of China

Full list of author information is available at the end of the article
}

important events in the establishment of parasite infections.

Calcium $\left(\mathrm{Ca}^{2+}\right)$ plays an important role in regulating parasite protein secretion, movement, differentiation, invasion and escape from host cells [2]. In eukaryotic cells, $\mathrm{Ca}^{2+}$ is an important signaling molecule, acting as a second messenger and regulating many physiological processes in the body [3]. When cells are stimulated by hormones or electricity, cytoplasmic $\mathrm{Ca}^{2+}$ concentrations increase, causing a series of intracellular physiological responses [4]. Calciumdependent protein kinases (CDPKs) are effectors of $\mathrm{Ca}^{2+}$ signaling that play important roles in cells. Recently, CDPKs have been found in plants, green algae and apicomplexan protozoans but have not been reported in bacteria, nematodes, fungi or vertebrates [5]. In cells, CDPKs 
phosphorylate substrate proteins to produce an amplification cascade reaction that transmits the $\mathrm{Ca}^{2+}$ signal. CDPKs have four domains: a variable region, a catalytic region, a link region, and a regulatory region. The catalytic zone can bind to ATP and serine or threonine residues of phosphorylated substrates. In the absence of $\mathrm{Ca}^{2+}$ ions, the linker region binds to the catalytic zone of the substrate and inhibits kinase activity. The regulatory region is a $\mathrm{Ca}^{2+}$ binding zone with EF chiral structure, which allows for CDPKs to be highly compatible with $\mathrm{Ca}^{2+}$ and not dependent on calmodulin [6].

In apicomplexan protozoans, CDPKs have been identified as part of the mechanistic link between $\mathrm{Ca}^{2+}$ signaling and differentiation, motility, invasion and escape from the host cell [2, 7]. Different CDPKs have specific expression patterns at different developmental stages and they regulate many $\mathrm{Ca}^{2+}$-dependent physiological processes. For example, in Plasmodium falciparum, PfCDPK1 can phosphorylate Myosin A tail domain-interacting protein (MTIP) and glideosomeassociated protein 45 (GAP45), which are the components of the motor complex that providing a driving force for parasites to invade the host [8]. The peptide P3, a part of the PfCDPK1 junction domain, inhibits the activity of CDPK1 and the secretion of microneme proteins during the invasion of erythrocytes by $P$. falciparum merozoites, indicating that CDPK1 is a key regulatory molecule during movement and invasion of host cells by asexual blood stage $P$. falciparum parasites [9]. Similar findings have been reported in Toxoplasma gondii. Conditional suppression of TgCDPK1 results in a block of essential phenotypes, including parasite motility and host cell invasion and escape, indicating that TgCDPK1 controls $\mathrm{Ca}^{2+}$-dependent secretion of microneme proteins [10]. TgCDPK1 exploits ATP-binding pockets to recognize its substrates, which include the dynamin-related protein DrpB [11]. TgCDPK7 knockdown parasites show significant growth defects and do not progress through cell division. Additionally, $T g \mathrm{CDPK} 7$ affects the partitioning and number of centrosomes during parasite division and the polarity of budding, which illustrates that $\mathrm{TgCDPK} 7$ is necessary to maintain the distribution and localization of centrosomes in T. gondii, an essential process for survival during the breeding stage [12].

Recent studies on E. tenella CDPK members have suggested that CDPKs regulate biological functions in $E$. tenella [13-15]. In a preliminary study of its function, it was found that EtCDPK3 was localized to the apical end of sporozoites during the initial invasion stage. Specific antibodies blocking EtCDPK3 inhibit host invasion, indicating that EtCDPK3 participates in host cell invasion and development within the host [16]. In our previous report, another E. tenella CDPK, EtCDPK4, was found to be highly expressed during the merozoite stage, although transcriptome levels of EtCDPK4 were highest during the sporozoite stage. Inhibiting the activity of $E t C D P K 4$ reduced sporozoite invasion, indicating that $E t$ CDPK4 participates in host cell invasion [15]. However, the $E t C D P K 4$ regulatory mechanisms and targets in E. tenella remain unclear.

Analyses of protein-protein interactions (PPIs) are crucial for the study of various cellular processes and protein function [17]. To further understand the function of EtCDPK4, we conducted yeast two-hybrid $(\mathrm{Y} 2 \mathrm{H})$ screening and identified an interaction between EtCDPK4 and E. tenella serine protease inhibitor 1 (EtSerpin). Moreover, we confirmed the interaction between EtCDPK4 and EtSerpin in DF-1 cells by coimmunoprecipitation (Co-IP) and bimolecular fluorescence complementation (BiFC).

\section{Methods}

\section{Antisera and recombinant plasmids}

The following antibodies used for immunoblotting and immunofluorescence assays (IFAs) were prepared and stored at $-20{ }^{\circ} \mathrm{C}$ in the laboratory: anti-EtCDPK4 rabbit and mouse antisera [15], anti-EtSerpin rabbit antisera are described elsewhere [18]. The recombinant plasmid pCAGGS-EtSerpin was a gift from Dr. Ye Wang, stored at the laboratory.

The sequences of primers used for PCR are provided in Table 1. To express fusion proteins, EtCDPK4 (ETH 00010685) was cloned into pcDNA3.1-flag (Biovector, Cambridge, MA, USA) and pBIFC-VC155 vectors and the recombinant plasmids were designated pCDNA3.1flag-EtCDPK4 and pBIFC-VC155-EtCDPK4. EtSerpin was cloned into the SalI and XhoI sites of pBIFC-VN155 using a ClonExpress kit (Vazyme, Nanjing, China).

\section{Parasite}

Eimeria tenella (CAAS211116-11) was obtained from the Shanghai Veterinary Research Institute, Chinese Academy of Agricultural Sciences. The parasite was

Table 1 Sequences of primers used in this study

\begin{tabular}{ll}
\hline Primer ID & Primer sequence (5'-3') \\
\hline pBiFC-VN155-Serpin UP & ATGGCCATGGAGGCCCGAATTCGGG \\
pBiFC-VN155-Serpin LOW & ACGCATGGCTCTGTTGAGTAAGCT \\
pBiFC-VC155-CDPK4 UP & GCGTGCTGCTGCCGG \\
pBiFC-VC155-CDPK4 LOW & GGTGATGGGTGGGGCGGGGGAGGGT \\
& GCGGTACCAAATTCGTCCCAGTCAA \\
pCDNA3.1-flag-CDPK4 UP & TCTGCCCAT \\
& GGGGATCCATGGAGCAGGTGATG \\
pCDNA3.1-flag-CDPK4 LOW & GCGAATTCCTAAAATTCGTCCCAG \\
& TCAATCTGCCCAT \\
\hline
\end{tabular}


obtained as previously described [19] by inoculation 2week-old chickens which were free of infection before experimental inoculation.

Unsporulated oocysts were obtained from the cecal contents of chickens infected with $1 \times 10^{4}$ E. tenella sporulated oocysts at 8 days post-infection (p.i.). Sporulated oocysts were harvested from the unsporulated oocysts which underwent sporulation with $2.5 \%$ potassium dichromate at $28{ }^{\circ} \mathrm{C}$ for $72-120 \mathrm{~h}$ in the presence of oxygen. Sporozoites were excysted with trypsin and chicken bile in vitro and purified from cleaned, sporulated oocysts by chromatography over columns of nylon wool and DE52 cellulose as previously described [19]. Secondgeneration merozoites (merozoites II) were isolated from the cecum and the cecal contents of chickens $115 \mathrm{~h}$ after infection E. tenella and then purified with Percoll [20].

\section{Construction of a Y2H cDNA library of E. tenella merozoites II}

Yeast strains Y187 and Y2H Gold (Takara, Tokyo, Japan) used for the $\mathrm{Y} 2 \mathrm{H}$ screen were prepared according to Yeastmaker $^{\text {TM }}$ Yeast Transformation System 2 User Manual (Clontech, Mountain View, CA, USA).

Total RNA was isolated from merozoites II of $E$. tenella with Trizol (Life Technologies, Carlsbad, CA, USA) according to the manufacturer's instructions. PolyA $^{+}$was purified with a PolyA ${ }^{+}$Tract mRNA Isolation System kit (Promega, Madison, WI, USA) after the quality of total RNA was assessed. The Y2H cDNA library of E. tenella merozoites was constructed using a Make Your Own "Mate \& Plate" Library System kit (Clontech, Mountain View, CA, USA). First-strand cDNA was synthesized and then amplified into doublestranded cDNAs (dscDNAs) using long distance PCR. dscDNAs shorter than 200 bp were removed using a Chroma Spin column (Takara, Tokyo, Japan). dscDNAs were cloned into the pGADT7-Rec vector (Clontech, Mountain View, CA, USA). The resulting plasmids were transformed into the Y187 yeast strain according to the instructions. To determine the transformation efficiency, $100 \mu \mathrm{l}$ of $1: 10,000,1: 1000,1: 100$ and $1: 10$ dilutions were spread on $100-\mathrm{mm}$ plates containing synthetic dropout (SD)/-Leu media and incubated at $30{ }^{\circ} \mathrm{C}$ for $3-5$ days. The remainder was spread on another SD/-Leu plate and the library solution was collected. To calculate the library size, $100 \mu \mathrm{l}$ of $1: 10,000,1: 1000,1: 100$ and $1: 10 \mathrm{di}-$ lutions of the library solution were spread onto media and incubated at $30{ }^{\circ} \mathrm{C}$ for 3-5 days. Fifty-one colonies were randomly selected for PCR identification and analysis of library insert size and recombination efficiency.

\section{$\mathrm{Y} 2 \mathrm{H}$ library screening}

EtCDPK4 cDNA was inserted in-frame with the GAL4 DNA-binding domain into the vector pGBKT7-GAL4 to construct the recombinant plasmid pGBKT7-EtCDPK4. A non-autoactivating and non-toxic pGBKT7-EtCDPK4 were acquired and bait proteins were expressed [21]. For interaction mating, the bait protein and the library were co-cultured at $30{ }^{\circ} \mathrm{C}$ with shaking for $20 \mathrm{~h}$ then spreading onto SD/-Leu/-Trp/-His/-Ade (QDO) selection media. After mating, clones were transferred to QDO media. Blue clones were confirmed by culturing on $\mathrm{SD} /$ Leu/-Trp/-His/-Ade supplemented with X- $\alpha$-gal (QDO/ $\mathrm{X})$ media and then cultured on SD/-Leu/-Trp/-His/-Ade supplemented with $\mathrm{X}$ - $\alpha$-gal and aureobasidin $\mathrm{A}(\mathrm{QDO} /$ $\mathrm{X} / \mathrm{A}$ ) media. Only those clones growing on $\mathrm{QDO} / \mathrm{X} / \mathrm{A}$ media were further characterized. Confirmation of interacting clones was performed by sequencing and nontarget plasmids were eliminated. Positive clones were further confirmed by prey plasmid rescue and retransformation into $\mathrm{Y} 2 \mathrm{H}$ Gold with pBDGAL4EtCDPK4 or with the negative control plasmid (empty pBD-GAL4).

\section{Immunolocalization}

Purified, freshly excysted sporozoites and merozoites II were incubated in phosphate-buffered saline (PBS), transferred to a glass slide, and air-dried as previously described [22]. The chicken embryo fibroblast (DF-1) cell line was cultured in Dulbecco's Modified Eagle's Medium (DMEM) (Gibco, Grand Island, NY, USA) supplemented with $10 \%$ fetal bovine serum (FBS) and $1 \%$ penicillin/streptomycin. $2.0 \times 10^{5}$ cells per well with slices were used for parasite invasion [18]. Purified sporozoites were washed three times with sterile PBS, infected into DF-1 cells then cultured at $41{ }^{\circ} \mathrm{C}$ for $2 \mathrm{~h}$. The cells cultured with slices were collected and washed with PBS. The slices were air-dried and fixed in $4 \%$ paraformaldehyde for $20 \mathrm{~min}$, then permeabilized with $0.1 \%$ Triton X-100 in PBS for $20 \mathrm{~min}$ and incubated with $2 \%$ bovine serum albumin (BSA) in PBS overnight at $4{ }^{\circ} \mathrm{C}$. At dilutions of EtCDPK 4 and EtSerpin antisera for $1 \mathrm{~h}$, a 1:500 dilution of goat anti-rabbit IgG fluorescein isothiocyanate (FITC)-conjugated antibody (Sigma, St. Louis, $\mathrm{MO}, \mathrm{USA}$ ) and goat anti-mouse IgG cyanine (Cy3)-conjugated antibody (Sigma, St. Louis, MO, USA) were added and incubated for $1 \mathrm{~h}$ at $37{ }^{\circ} \mathrm{C}$. 4,6-diamidino-2phenylindole $(10 \mu \mathrm{g} / \mathrm{ml}$, Beyotime, Haimen, China) was used to stain nuclei for $30 \mathrm{~min}$. After each step, slides were washed three times with PBS. $50 \mu$ l Fluoromount Aqueous Mounting Medium (Sigma, St. Louis, MO, USA) was added before observation under a fluorescence microscope (Olympus, Tokyo, Japan).

\section{BiFC assay}

For BiFC assays, cells must take up the expression vector. Therefore, IFAs were performed to confirm expression of the fusion proteins in the DF-1 cells. The 
recombinant plasmids VC155-EtCDPK4 and VN155EtSerpin were transfected into $6.0 \times 10^{5} \mathrm{DF}-1$ cells and cultured in six-well plates for $24 \mathrm{~h}$. Briefly, $4 \mu \mathrm{g}$ plasmid DNA and $10 \mu \mathrm{l}$ lipofectamine 2000 (Invitrogen, Carlsbad, CA, USA) were mixed, incubated at room temperature for $20 \mathrm{~min}$, and gently added to the cells. After $6 \mathrm{~h}$, the DNA transfection reagent was replaced with DMEM containing $2 \% \mathrm{FBS}$ and $200 \mathrm{U} / \mathrm{ml}$ penicillin/streptomycin. For IFAs, DF-1 cells transfected with recombinant plasmids were fixed in $2 \%$ paraformaldehyde for $20 \mathrm{~min}$. The samples were permeabilized with $0.1 \%$ Triton $\mathrm{X}-100$ in PBS pH7.4 for 20 min and then blocked with $2 \%$ BSA in PBS at $4{ }^{\circ} \mathrm{C}$ overnight. The relevant antisera and the goat anti-rabbit secondary antibodies were used for incubation. Finally, the samples were visualized using a fluorescence microscope.

After confirming that the DF-1 cells could take up the two constructs, the recombinant plasmids VC155EtCDPK4 and VN155-EtSerpin were co-transfected into DF-1 cells. $30 \mathrm{~h}$ later, the cells were observed under an inverted fluorescence microscope. Two non-fluorescent fragments, pBiFC-bfosVC155 and pBiFC-bjunVN155, form a fluorescent complex that can be detected using a fluorescence microscope were used as positive controls. Two nonfluorescent fragments, pBiFC-bfosVC155 (delta ZIP) and pBiFC-bjunVN155, were used as negative controls.

\section{Co-IP}

To confirm their expression in DF-1 cells, the recombinant plasmids pCDNA3.1-flag-EtCDPK4 and pCAGGSEtSerpin were transfected into $2.0 \times 10^{6} \mathrm{DF}-1$ cells and analyzed by western blot. After confirming that the DF-1 cells could take up the two constructs, the recombinant plasmids were co-transfected into cells as described above. pCDNA3.1-flag and flag-EtCDPK4 were used as controls. After $48 \mathrm{~h}$, the transfected cells were washed twice with PBS and lysed with RAPI buffer, cell debris was removed by centrifugation at $12,000 \times \mathrm{rpm}$ for 10 min. The Co-IP assay was performed using the Pierce Co-Immunoprecipitation kit (Thermo, Waltham, MA, USA) following the manufacturer's instructions using antisera to EtCDPK4 for coupling to the resin. Samples were analysed by SDS-PAGE followed by Western blotting then detection with EtSerpin antisera or anti-flag antibody. The control was incubated with anti-Flag antibody only. The nitrocellulose membranes were incubated with anti-mouse fluorescent secondary antibody for anti-Flag and anti-rabbit fluorescent secondary antibody for EtSerpin antibodies.

\section{Results}

Eimeria tenella merozoite II $\mathrm{Y} 2 \mathrm{H}$ cDNA library construction Merozoite II cDNA from E. tenella was cloned into the pGADT7-rec vector. The resulting plasmids were transformed into the Y187 strain and spread onto SD/-Leu plates. Random clones were selected for PCR analysis (Fig. 1). Results showed that $93.2 \%$ of recombinants carried DNA sequences with an average length of $500 \mathrm{bp}$ from $E$. tenella merozoites. The size of the library was 9 . $6 \times 10^{9} \mathrm{CFU}$ and the transformation efficiency of the library was $4.1 \times 10^{5} \mathrm{cfu} / \mu \mathrm{g}$ pGADT7-Rec, which was sufficient for subsequent $\mathrm{Y} 2 \mathrm{H}$ screening.

\section{Y2H screening for proteins interacting with EtCDPK4}

$\mathrm{Y} 2 \mathrm{H}$ screening of the E. tenella merozoite II cDNA library with $E t$ CDPK4 as bait resulted in 69 blue colonies formed (Fig. 2a). Comparison of the DNA sequence of the positive plasmids with the genome of E. tenella (http://www.genedb.org) showed that 30 different $E$. tenella proteins were represented. To test whether these proteins interacted with $E t \mathrm{CDPK} 4$ in yeast, the positive plasmids were transformed into $\mathrm{Y} 2 \mathrm{H}$ Gold using pGADT7-EtCDPK4 and

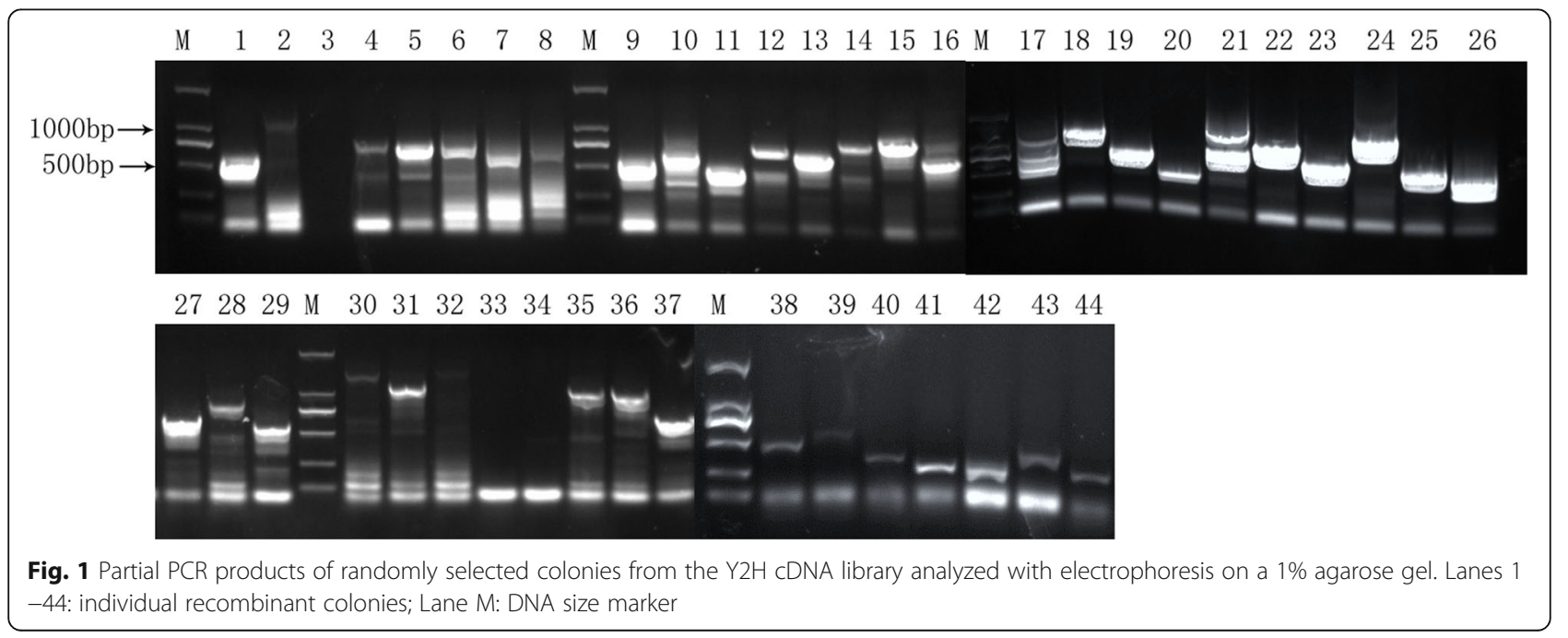


a
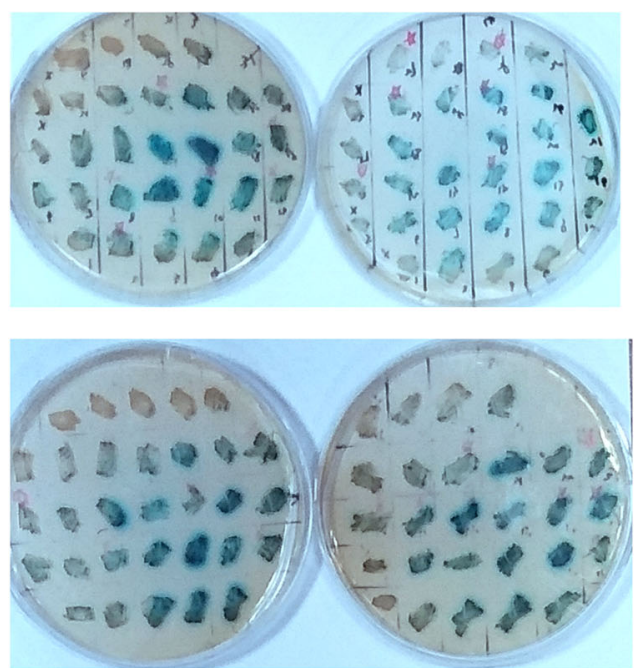

positive

b control

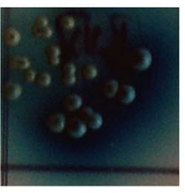

negative

control

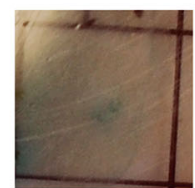

1

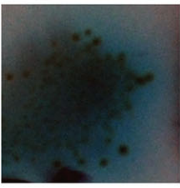

5

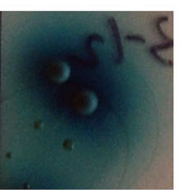

2

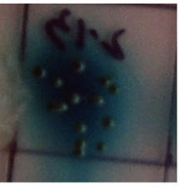

6

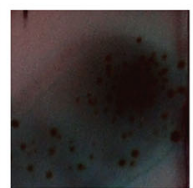

3

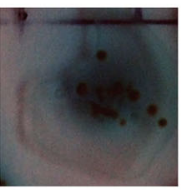

7

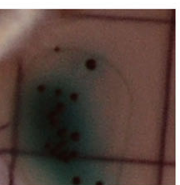

4

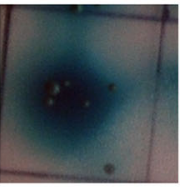

8

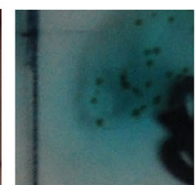

Fig. 2 Y $2 \mathrm{H}$ screen for proteins that interact with EtCDPK4. a Blue clones acquired by culturing on QDO/X/A media. b Positive plasmids confirmed by transforming Y2HGold with pGADT7-EtCDPK4

spread onto QDO/X/A plates. In total, eight blue colonies (Table 2) formed that contained proteins that interacted with EtCDPK4 in the Y2H system (Fig. 2b).

\section{Co-localization of EtCDPK4 and EtSerpin}

IFAs were performed to determine the location of EtCDPK4 and EtSerpin. Sporozoites and merozoites II treated with antiEtCDPK4 mouse antisera and anti-EtSerpin rabbit antisera showed EtCDPK4 and EtSerpin distributed throughout the cytoplasm (Fig. 3a, c). At 2 h p.i. of DF-1 cells by sporozoites both proteins of EtCDPK4 and EtSerpin were at the apical end (Fig. 3b). The co-localization indicated that the proteins of EtCDPK4 and EtSerpin function in the same location.

\section{Interaction between EtCDPK4 and EtSerpin assessed by}

BiFC

Expression of the plasmids VC155-EtCDPK4 and VN155-EtSerpin in DF-1 cells was confirmed using IFA
Table 2 EtCDPK4 targets identified by $\mathrm{Y} 2 \mathrm{H}$ screening

\begin{tabular}{lll}
\hline Gene ID & Annotation & MW (kDa) \\
\hline ETH_00011330 & SERPIN1 protein & 45.5 \\
ETH_00024500 & Hypothetical protein & 92.3 \\
ETH_00018145 & Hypothetical protein & 17.9 \\
ETH_00002065 & Hypothetical protein & 38.4 \\
ETH_00009380 & DNA-directed RNA polymerases I & 36.4 \\
& and III subunit RPAC1 & \\
EMH_00033980 & Hypothetical protein & 44.1 \\
ETH_00021190 & Sec63 domain-containing DEAD/DEAH & 246.7 \\
& box helicase & 58.0 \\
\hline ETH_00007745 & apical membrane antigen-1 \\
\hline
\end{tabular}




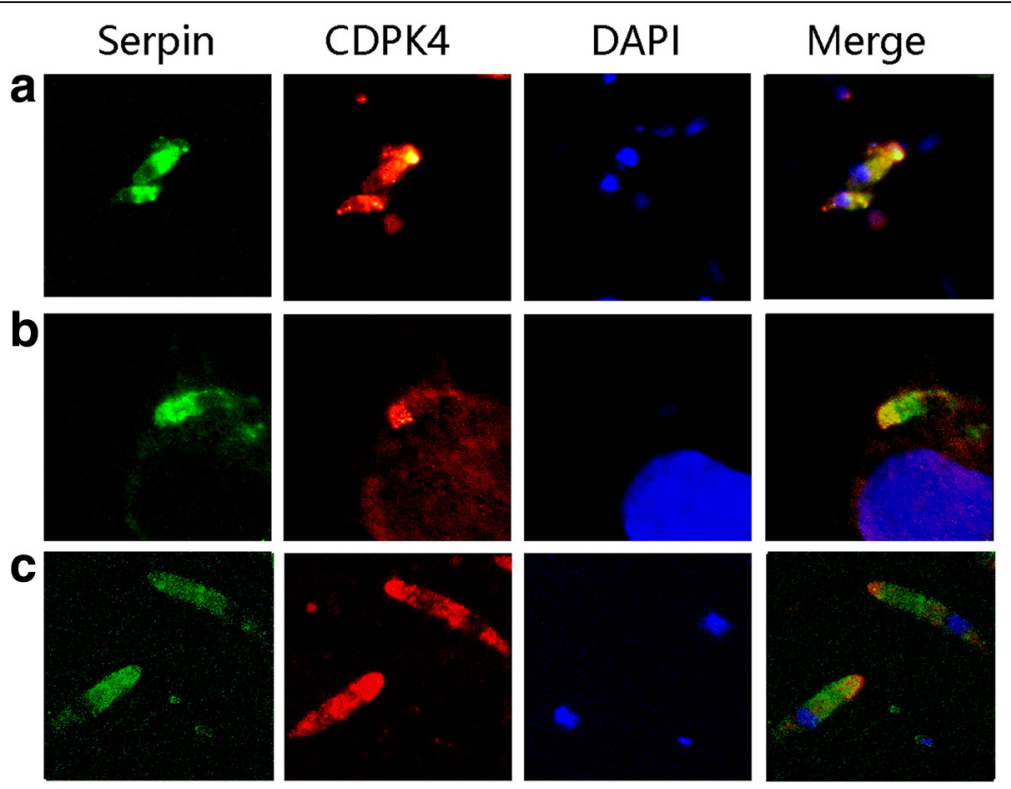

Fig. 3 Co-localization of EtCDPK4 and EtSerpin. a IFA performed using antisera against EtCDPK4 and EtSerpin in sporozoites. b IFA performed using antisera against EtCDPK4 and EtSerpin at 2h PI. c IFA performed using antisera against EtCDPK4 and EtSerpin in merozoites II . EtSerpin antibody reactivity was detected with FITC and anti-EtCDPK4 reactivity with Cy3 conjugated secondary antibodies

(data not shown). The recombinant plasmids VC155EtCDPK4 and VN155-EtSerpin were then co-transfected into DF-1 cells and observed with an inverted fluorescence microscope. When the positive controls bFos and bJun were transiently co-expressed in DF-1 cells, a positive BiFC signal was detected in the cells. A similar positive BiFC signal was detected when VC155EtCDPK4 and VN155-EtSerpin were transiently coexpressed in DF-1 cells, indicating that EtCDPK4 and EtSerpin interact in DF-1 cells (Fig. 4). In contrast, the

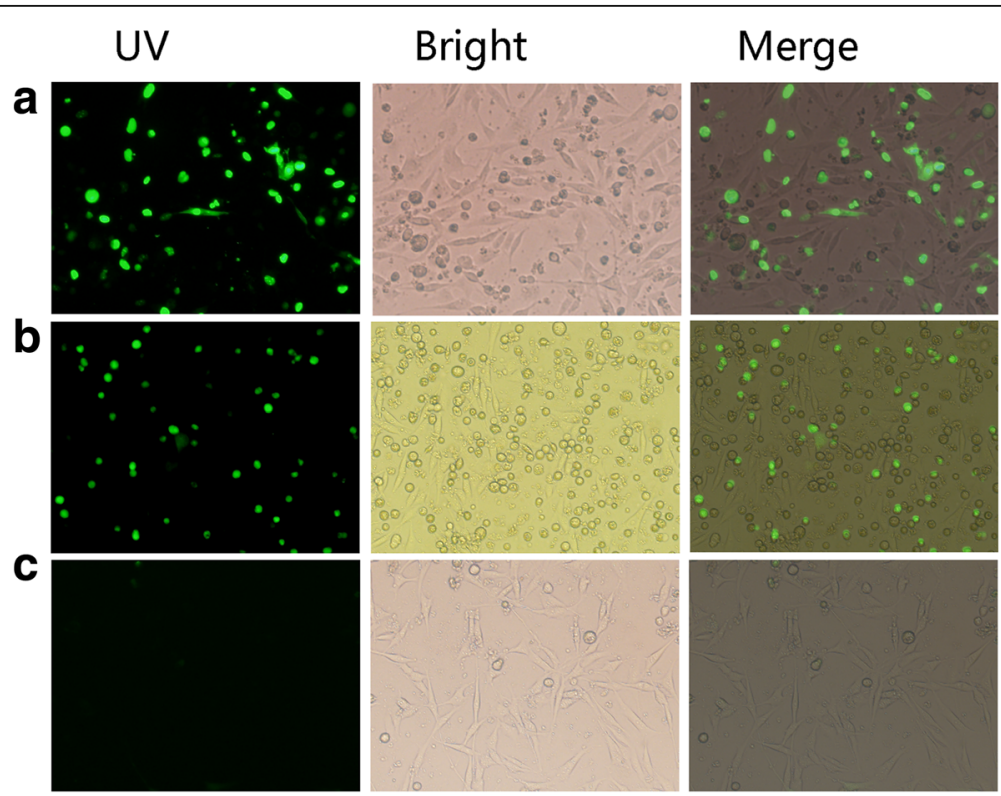

Fig. 4 Interaction between EtCDPK4 and EtSerpin in DF-1 cells assessed by BiFC. VC155-EtCDPK4 and VN155-EtSerpin were co-transfected into DF-1 cells. a Positive controls bFos and bJun co-transfected into DF-1 cells. b EtCDPK4 and EtSerpin co-transfected into DF-1 cells. c Negative controls bFos (delta ZIP) and bJun co-transfected into DF-1 cells 
negative controls bFos (delta ZIP) and bJun did not produce a detectable fluorescent signal.

\section{Interaction between EtCDPK4 and EtSerpin assessed by Co-IP}

Western-blotting results showed that the fusion protein flag-EtCDPK4 and EtSerpin were successfully expressed in DF-1 cells (Fig. 5a). Co-IP assays showed that when flag-EtCDPK4 and EtSerpin proteins were incubated with resin that was covalently coupled with EtCDPK4 antisera, EtSerpin was eluted with EtCDPK4 from the resin by elution buffer (Fig. 5b). In contrast, when pCDNA3.1-flag was used instead of EtSerpin in control experiments, EtCDPK4 alone was detected in the eluate (Fig. 5b). Based on these data, we conclude that an interaction exists between $E t C D P K 4$ and EtSerpin.

\section{Discussion}

Biochemical analysis of protein complexes and identification of their components is fundamental to the understanding of protein function [23, 24]. Currently, several methods for identifying protein interactions exist, including $\mathrm{Y} 2 \mathrm{H}$ techniques, Co-IP, BiFC, phage display technology and pull down experiments.

In the present study, we used the $\mathrm{Y} 2 \mathrm{H}$ technique to screen for proteins that interact with EtCDPK4. High quality libraries are one of the key elements of $\mathrm{Y} 2 \mathrm{H}$ screens. In this study, a high quality Y2H cDNA library was constructed using E. tenella merozoites II We achieved a recombination rate of $93.2 \%$ and a library size of $9.6 \times 10^{-9} \mathrm{CFU}$, which was sufficient for subsequent $\mathrm{Y} 2 \mathrm{H}$ screening. A total of eight interacting proteins were identified (Table 2), one of which was previously reported and described as Serpin1 (ETH_00011330) in our lab [18]. Only eight positive interactions were confirmed on a second round screening. There are maybe several reasons: (i) the incorrect folding and/or instability of an $\mathrm{AD}$ fusion protein that could interact with its interacting partner, there are maybe some of these $\mathrm{AD}$ fusion proteins in the E. tenella merozoites II $\mathrm{Y} 2 \mathrm{H}$ cDNA library; (ii) the toxicity of some fusion proteins that could affect the viability of transformed cells [25]; (iii) the quality of the library is a key parameter for the success of a screening, although the quality of E. tenella merozoites II Y2H cDNA library that we constructed is good, it cannot include all the cDNAs. Some mRNAs encoding putative interacting proteins are expressed at relatively low levels; these proteins might not be identified. In addition there were several identified plasmids $(7 / 30)$ failed to rescue so there may well be other proteins which interacted with EtCDPK4. In future study, we will screen the putative interacting proteins using other methods. The $\mathrm{Y} 2 \mathrm{H}$ system may have technical or biological false positives like any assay system [26]. Therefore, we used other methods, including Co-IP, BiFC and co-localization, to further verify the interaction between EtCDPK4 and EtSerpin.

Detection of PPIs in living cells is particularly important for understanding biological process [27, 28]. One of effective ways for studying PPIs is BiFC [29]. This assay offers several advantages over other techniques such as $\mathrm{Y} 2 \mathrm{H}$. The method enables real-time observation of PPIs in their natural environment, such as in live cells or animals [30, 31]. In addition, the subcellular localization of the PPI can be observed directly from BiFC [31]. So in this study, we used the BiFC to verify the interaction between EtCDPK4 and EtSerpin.

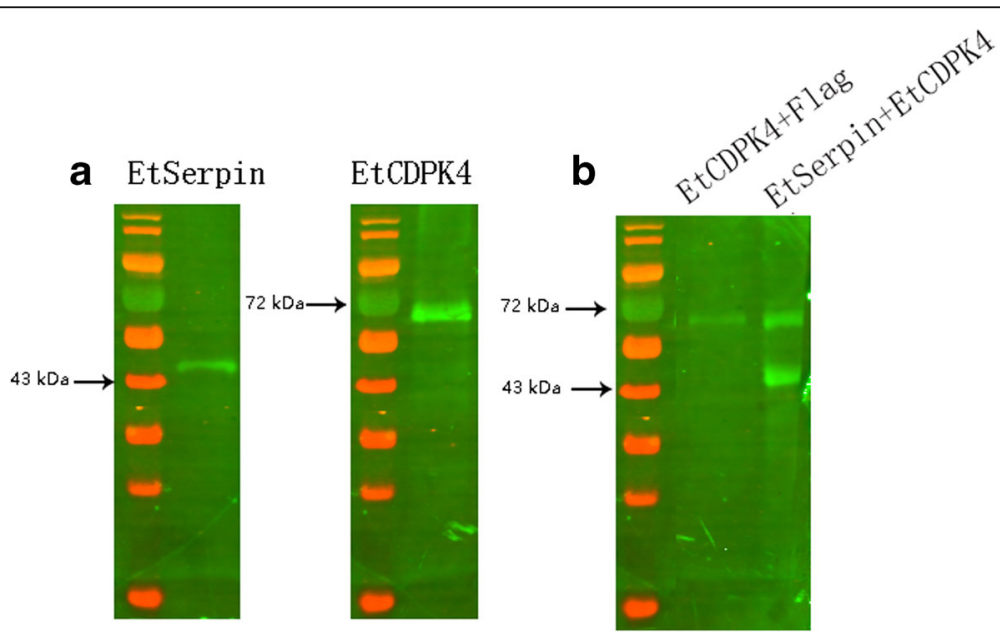

Fig. 5 Interaction between EtCDPK4 and EtSerpin assessed with Co-IP. a DF-1 cells were transfected with pCDNA3.1-flag-EtCDPK4 and pCAGGSEtSerpin and cellular lysates were analyzed with immunoblotting with antisera against EtCDPK4 and EtSerpin. b The Co-IP was performed using immobilised antisera against EtCDPK4. Detection of eluted proteins on immunoblots was by EtSerpin and/or anti-flag for EtCDPK4 antibodies 
CDPKs are present in plants, algae, ciliates and apicomplexan parasites. In plants, CDPKs regulate plant development and biotic and abiotic stress responses. The N-terminal domain of CDPK plays a key role in subcellular localization and function [32]. Most CDPKs have myristoylation sites and cysteine residues that allow for N-terminal palmitoylation and contribute to the localization of CDPKs. Arabidopsis thaliana AtCPK16 is predicted to be localized to the chloroplast based on multiple prediction methods, whereas N-terminal acylation at $\mathrm{N}$-myristoylation and palmitoylation sites prevents localization to the chloroplast [33]. The $\mathrm{N}$ terminus not only determines subcellular localization, but also interacts with target proteins. For example, $\mathrm{Ni}$ cotiana tabacum NtCDPK1 could phosphorylate the basic leucine zipper transcription factor RSG (repression of shoot growth) in tobacco. A chimeric CDPK containing NtCDPK1 N-terminus fused to AtCPK9 can also phosphorylate and interact with RSG, although native AtCPK9 can neither bind nor phosphorylate RSG [34]. Many Arabidopsis CDPKs are membrane localized or membrane associated, which is mediated by N-terminal acylation [35]. In $T$. gondii, the substrate of $T g C D P K 1$, DrpB, interacts with CDPK1 at the N-terminal ATPbinding pocket [11]. In P. falciparum, PfCDPK7 interacts with phosphatidylinositol 4,5-bisphosphate via its pleckstrin homology domain, guiding its subcellular localization [36]. Functional structure prediction indicates that EtCDPK4 contains three $\mathrm{N}$-myristoylation sites, an ATP binding domain, and a serine/threonine protein kinase activation site [15]. N-myristoylation sites and ATP binding domain contribute to the subcellular localization and functions of EtCDPK4, which include $\mathrm{Ca}^{2+}$ signaling and interacting with substrate proteins. We hypothesise that EtCDPK4 and EtSerpin interact through the ATP binding domain and play a role in sporozoite invasion. The $\mathrm{N}$-terminal myristolation site of EtCDPK4 may help the complex of EtCDPK4 and EtSerpin to locate to the apex near the membrane surface of parasites when sporozoites invade host cells.

EtSerpin was one of the putative interacting proteins of EtCDPK4. Serine protease inhibitors (serpins) are a class of proteins composed of 300-500 amino acids with a molecular weight between $40-60 \mathrm{kDa}$. Although intracellular serpins have been reported, most serpins are present in the extracellular environment [37-39]. Over 500 members of the serpin superfamily have been identified in animals, plants, bacteria, archaea and viruses [40]. In mammals, serpins play crucial roles in processes such as blood coagulation and fibrinolysis [38, 41]. Most serpins consist of three $\beta$-folds and $8-9 \alpha$-helices. The typical serpin structure includes an exposed reactive center loop conformation above the body of the molecule [42]. Most serpins undergo a significant conformational change from the stressed to the relaxed state that can result in inhibition of target proteases. The activity of some small protease inhibitors can be regulated by specific factors. For example, SERPINC1 is a rare inhibitor that inhibits factor Xa with the cofactor heparin in human [43]. SERPINC1, protease and heparin form a stable ternary complex. Therefore, synergistic interactions between serpins and other molecules can result in different roles for serpin proteins. In the case of protein $\mathrm{Z}$ dependent protease inhibitors, protein $\mathrm{Z}$ as a vitamin $\mathrm{K}$-dependent co-factors to promote the inhibitory activity of the serpin with FXa on negatively charged phospholipid vesicles and calcium [44]. Vaspin is visceral adipose tissue-derived serine protease inhibitor, promoted the phosphorylation of Akt through PI3K signaling pathway [45]. In parasites, Serpin plays an important role in the inflammatory response, regulating host immunity, development and anticoagulation. For example, Ixodes ricinus salivary serpin has anticoagulant activity, including coagulation and fibrinolysis inhibition and binds to cells/macrophages and inhibits TNF secretion [46]. In T. gondii, SERPIN B3 and B4 act via STAT6 activation to inhibit casapase 3 , PARP activation, and DNA fragmentation [47]. In this study, $\mathrm{Y} 2 \mathrm{H}, \mathrm{Co}-\mathrm{IP}$, and BiFC were used to identify an interaction between EtCDPK4 and EtSerpin. We expect that, EtCDPK4 may interact with EtSerpin as a cofactor, similar to SERPINC1 or protein Z, the interaction between EtSerpin and EtCDPK4 may enhance protease inhibitory activity of EtSerpin during sporozoites invasion into host cells. At the same time, the phosphorylation of EtCDPK4 may be elevated by EtSerpin.

In this study, EtCDPK4 and EtSerpin were located mainly in the cytoplasm of sporozoites and merozoites II. Co-localization experiments showed that EtCDPK4 and EtSerpin shared the same apical location during the early invasion of sporozoites into DF-1 cells. These results are consistent with previous reports [15, 18]. Another EtSerpin has been reported to be detected 24 h p.i. in DF-1 cells in vitro [18]. Additionally, EtCDPK4 has been detected in the vacuole $12 \mathrm{~h}$ p.i. [15]. In vitro, sporozoite invasion inhibition assays indicated that polyclonal antibodies against these two proteins can also reduce the ability of E. tenella sporozoites to invade host cells $[15,18]$. Therefore, we speculate that the interaction between EtCDPK4 and EtSerpin is likely to play an important role in sporozoite invasion. When the sporozoites invade the host cells, the complex could release into the host cells to inhibit host protease activity which may delay host cell apoptosis.

Although we confirmed the interaction between EtSerpin and EtCDPK4 with several methods, we also identified but did not confirm other proteins that interact with EtCDPK4 using the $\mathrm{Y} 2 \mathrm{H}$ screen. The interaction 
between EtSerpin and EtCDPK4 may contribute to the invasion of E. tenella in host cells, the complex could inhibit host protease activity to delay host cell apoptosis during sporozoite development in host cells. However, further research on the function of the interaction between EtSerpin and EtCDPK4 at the time of invasion is needed.

\section{Conclusions}

In this study, we constructed a $\mathrm{Y} 2 \mathrm{H}$ cDNA library to screen for proteins that interact with EtCDPK4. EtSerpin was demonstrated to co-localize and interact with $E t C D P K 4$, which may promote to the invasion and development of E. tenella in host cells.

\begin{abstract}
Abbreviations
AtCPK9: Arabidopsis thaliana CPK9; BiFC: Bimolecular fluorescence complementation; BSA: Bovine serum albumin; CDPKs: Calcium dependent protein kinase; Co-IP: Co-immunoprecipitation; DMEM: Dulbecco's Modified Eagle's Medium; dscDNAs: Double-stranded cDNAs; EtCDPK3: Eimeria tenella CDPK3: EtCDPK4: Eimeria tenella CDPK4; EtSerpin: Eimeria tenella Serpin; FBS: Fetal bovine serum; GAP45: Glideosome-associated protein 45; IFAs: Immunofluorescence assays; MTIP: Myosin A tail domain-interacting protein; NtCDPK1: Nicotiana tabacum CDPK1; PBS: Phosphate-buffered saline; PfCDPK1: Plasmodium falciparum CDPK1; p.i.: Post-infection; PPI: Proteinprotein interactions; QDO: SD/-Leu/-Trp/-His/-Ade; QDO/X: SD/-Leu/-Trp/His/-Ade supplemented with X-a-gal; QDO/X/A: SD/-Leu/-Trp/-His/-Ade supplemented with $\mathrm{X}$-a-gal and aureobasidin A; TgCDPK1: Toxoplasma gondii. CDPK1; YPDA: Yeast peptone dextrose adenine; Y2H: Yeast two-hybrid
\end{abstract}

\section{Acknowledgments}

We would like to thank Professor Chan Ding (CAAS, Beijing, China) for generously providing the DF-1 cell line used in this study.

\section{Funding}

This work was supported by the National Natural Science Foundation of China (Grant Nos. 31572266 and 31272557) and Central Public Welfare Research Institutions and Basic Scientific Research Business Expenses (No. 2016JB10) and Shanghai Minhang District talent development special funds.

\section{Authors' contributions}

$\mathrm{HYH}$ and $\mathrm{BH}$ conceived and designed the study. $\mathrm{LL}, \mathrm{BH}$ and $\mathrm{QPZ}$ performed the experiments. $\mathrm{HD}$ and $\mathrm{SHZ}$ analyzed the data. $\mathrm{LL}$ and $\mathrm{QPZ}$ collected parasites. LL and HYH wrote the manuscript. All authors read and approved the final manuscript.

\section{Ethics approval}

The protocol was approved and authorized by the Animal Care and Use Committee of the Shanghai Veterinary Research Institute, Chinese Academy of Agricultural Sciences.

\section{Competing interests}

The authors declare that they have no competing interests.

\section{Publisher's Note}

Springer Nature remains neutral with regard to jurisdictional claims in published maps and institutional affiliations.

\section{Author details}

${ }^{1}$ College of Life and Environment Sciences, Shanghai Normal University, Shanghai 200234, China. ${ }^{2}$ Shanghai Veterinary Research Institute, Chinese Academy of Agricultural Sciences, Key Laboratory of Animal Parasitology of Ministry of Agriculture, Minhang, Shanghai 200241, People's Republic of China.
Received: 6 December 2017 Accepted: 13 April 2018

Published online: 23 April 2018

\section{References}

1. Santos JM, Soldati-Favre D. Invasion factors are coupled to key signaling events leading to the establishment of infection in apicomplexan parasites. Cell Microbiol. 2011:13:787-96.

2. Billker O, Lourido S, Sibley LD. Calcium-dependent signaling and kinases in apicomplexan parasites. Cell Host Microbe. 2009;5:612-22.

3. Hetherington $\mathrm{AM}$, Brownlee $\mathrm{C}$. The generation of $\mathrm{Ca}^{2+}$ signals in plants. Annu Rev Plant Biol. 2004:55:401-27.

4. Harper JF, Harmon A. Plants, symbiosis and parasites: a calcium signaling connection. Nat Rev Mol Cell Biol. 2005:6:555-66.

5. Hamel LP, Sheen J, Seguin A. Ancient signals: comparative genomics of green plant CDPKs. Trends Plant Sci. 2014;19:79-89.

6. Wan B, Lin Y, Mou T. Expression of rice $\mathrm{Ca}^{2+}$-dependent protein kinases (CDPKs) genes under different environmental stresses. FEBS Lett. 2007;581: 1179-89.

7. Moreno SNJ, Ayong L, Pace DA. Calcium storage and function in apicomplexan parasites. Essays Biochem. 2011:51:97-110.

8. Green J, Rees-Channer R, Howell S, Martin S, Knuepfer E, Taylor H, et al. The motor complex of Plasmodium falciparum: phosphorylation by a calciumdependent protein kinase. J Biol Chem. 2008;283:30980-9.

9. Bansal A, Singh S, More KR, Hans D, Nangalia K, Yogavel M, et al. Characterization of Plasmodium falciparum calcium-dependent protein kinase 1 (PfCDPK1) and its role in microneme secretion during erythrocyte invasion. J Biol Chem. 2013;288:1590-602.

10. Lourido S, Shuman J, Zhang C, Shokat KM, Hui R, Sibley LD. Calciumdependent protein kinase 1 is an essential regulator of exocytosis in Toxoplasma. Nature. 2010;465:359-62

11. Lourido S, Jeschke GR, Turk BE, Sibley LD. Exploiting the unique atp-binding pocket of Toxoplasma calcium-dependent protein kinase 1 to identify its substrates. ACS Chem Biol. 2013:8:1155-62

12. Morlon-Guyot J, Berry L, Chen CT, Gubbels MJ, Lebrun M, Daher W. The Toxoplasma gondii calcium-dependent protein kinase 7 is involved in early steps of parasite division and is crucial for parasite survival. Cell Microbiol. 2014;16:95-114.

13. Dunn PP, Bumstead JM, Tomley FM. Sequence, expression and localization of calmodulin-domain protein kinases in Eimeria tenella and Eimeria maxima. Parasitology. 1996;113:439-48.

14. Han HY, Lin JJ, Zhao QP, Hui D, Jiang LL, Xu MQ, et al. Identification of differentially expressed genes in early stages of Eimeria tenella by suppression subtractive hybridization and cDNA microarray. J Parasitol. 2010:96:95-102

15. Wang Z, Bing H, Hui D, Zhao Q, Zhu S, Xia W, et al. Molecular characterization and functional analysis of a novel calcium-dependent protein kinase 4 from Eimeria tenella. PLoS One. 2016;11:e0168132.

16. Han HY, Zhu SH, Jiang LL, Li Y, Dong H, Zhao OP, et al. Molecular characterization and analysis of a novel calcium-dependent protein kinase from Eimeria tenella. Parasitology. 2013;140:746-55.

17. Weinthal D, Tzfira T. Imaging protein-protein interactions in plant cells by bimolecular fluorescence complementation assay. Trends Plant Sci. 2009;14: $59-63$.

18. Jiang L, Lin J, Han H, Zhao Q, Dong H, Zhu S, et al. Identification and partial characterization of a serine protease inhibitor (serpin) of Eimeria tenella. Parasitol Res. 2012;110:865-74.

19. Tomley F. Techniques for isolation and characterization of apical organelles from Eimeria tenella sporozoites. Methods. 1997;13:171-6.

20. Geysen J, Ausma J, Vanden BH. Simultaneous purification of merozoites and schizonts of Eimeria tenella (Apicomplexa) by Percoll flotation and assessment of cell viability with a double fluorescent dye assay. J Parasitol. 1991;77:989-93

21. Wang ZW, Dong H, Zhao QP, Xia WL, Zhu SH, Men QF, et al. Detection and construction of Eimeria tenella CDPK4 bait plasmid for yeast two-hybrid system. Chin J Anim Infect Dis. 2016;24:62-9.

22. Peroval M, Pery P, Labbe M. The heat shock protein 90 of Eimeria tenella is essential for invasion of host cell and schizont growth. Int J Parasitol. 2006; 36:1205-15.

23. Gavin AC, Bosche M, Krause R, Grandi P, Marzioch M, Bauer A, et al. Functional organization of the yeast proteome by systematic analysis of protein complexes. Nature. 2002;415:141-7. 
24. Ho Y, Gruhler A, Heilbut A, Bader GD, Moore L, Adams SL, et al. Systematic identification of protein complexes in Saccharomyces cerevisiae by mass spectrometry. Nature. 2002;415:180-3.

25. Ferro E, Baldini E, Trabalzini L. Use of the yeast two-hybrid technology to isolate molecular interactions of Ras GTPases. Methods Mol Biol. 2014;1120: 97-120.

26. Rajagopala SV. Mapping the protein-protein interactome networks using yeast two-hybrid screens. Adv Exp Med Bio. 2015;883:187-214.

27. Remy I, Michnick SW. Application of protein-fragment complementation assays in cell biology. BioTechniques. 2007;42:137-45.

28. Gandhi TK, Zhong J, Mathivanan S, Karthick L, Chandrika KN, Mohan SS, et al. Analysis of the human protein interactome and comparison with yeast, worm and fly interaction datasets. Nat Genet. 2006;38:285-93.

29. Pham CD. Detection of protein-protein interaction using bimolecular fluorescence complementation assay. Methods Mol Biol. 2015;1278:483-95.

30. Kerppola TK. Design and implementation of bimolecular fluorescence complementation (BiFC) assays for the visualization of protein interactions in living cells. Nat Protoc. 2006;1:1278-86.

31. Michnick SW. Protein fragment complementation strategies for biochemical network mapping. Curr Opin Biotechnol. 2003;14:610-7.

32. Dammann C, Ichida A, Hong B, Romanowsky SM, Hrabak EM, Harmon AC, et al. Subcellular targeting of nine calcium-dependent protein kinase isoforms from Arabidopsis. Plant Physiol. 2003;132:1840-8.

33. Stael S, Bayer RG, Mehlmer N, Teige M. Protein N-acylation overrides differing targeting signals. FEBS Lett. 2011;585:517-22.

34. Ito T, Nakata M, Fukazawa J, Ishida S, Takahashi Y. Alteration of substrate specificity: the variable $\mathrm{N}$-terminal domain of tobacco $\mathrm{Ca}_{2}^{+}$-dependent protein kinase is important for substrate recognition. Plant Cell. 2010;22: 1592-604.

35. Cheng SH, Sheen J. Calcium signaling through protein kinases. The Arabidopsis calcium-dependent protein kinase gene family. Plant Physiol. 2002;129:469-85.

36. Kumar P, Tripathi A, Ranjan R, Halbert J, Gilberger T, Doerig C, et al. Regulation of Plasmodium falciparum development by calcium-dependent protein kinase 7 (PfCDPK7). J Biol Chem. 2014;289:20386-95

37. Valdivieso E, Perteguer MJ, Hurtado C, Campioli P, Rodríguez E, Saborido A, et al. ANISERP: a new serpin from the parasite Anisakis simplex. Parasit Vectors. 2015;8:399.

38. Silverman GA, Bird Pl, Carrell RW, Church FC, Coughlin PB, Gettins PGW, et al. The serpins are an expanding superfamily of structurally similar but functionally diverse proteins evolution, mechanism of inhibition, novel functions, and a revised nomenclature. J Biol Chem. 2001;276:33293-6.

39. Gettins PGW. Serpin structure, mechanism, and function. Chem Rev. 2002; 102:4751-804

40. Irving JA, Pike RN, Lesk AM, Whisstock JC. Phylogeny of the serpin superfamily: implications of patterns of amino acid conservation for structure and function. Genome Res. 2000;10:1845-64.

41. Rau JC, Beaulieu LM, Huntington JA, Church FC. Serpins in thrombosis, hemostasis and fibrinolysis. J Thromb Haemost. 2007;5(Suppl. 1):102-15.

42. Law RH, Zhang Q, Mcgowan S, Buckle AM, Silverman GA, Wong W, et al. An overview of the serpin superfamily. Genome Biol. 2006;7:216.

43. Rezaie AR. Calcium enhances heparin catalysis of the antithrombin-factor Xa reaction by a template mechanism. Evidence that calcium alleviates Gla domain antagonism of heparin binding to factor Xa. J Biol Chem. 1998;273: 16824-7.

44. Tabatabai A, Fiehler R, Bronze GJ Jr. Protein Z circulates in plasma in a complex with protein Z-dependent protease inhibitor. Thromb Haemost. 2001;85:655-60.

45. Liu S, Li X, Wu Y, Duan R, Zhang J, Du F, et al. Effects of vaspin on pancreatic $\beta$ cell secretion via PI3K/Akt and NF-KB signaling pathways. PLoS One. 2017;12:e189722.

46. Prevot PP, Beschin A, Lins L, Beaufays J, Grosjean A, Bruys L, et al. Exosites mediate the anti-inflammatory effects of a multifunctional serpin from the saliva of the tick /xodes ricinus. FEBS J. 2009;276:3235-46.

47. Song KJ, Ahn HJ, Nam HW. Anti-apoptotic effects of SERPIN B3 and B4 via STAT6 activation in macrophages after infection with Toxoplasma gondii. Korean J Parasitol. 2012;50:1-6.

\section{Ready to submit your research? Choose BMC and benefit from:}

- fast, convenient online submission

- thorough peer review by experienced researchers in your field

- rapid publication on acceptance

- support for research data, including large and complex data types

- gold Open Access which fosters wider collaboration and increased citations

- maximum visibility for your research: over $100 \mathrm{M}$ website views per year

At BMC, research is always in progress.

Learn more biomedcentral.com/submissions 